\title{
Interação do regime hídrico com as relações nutricionais em ecossistema manguezal
}

\author{
Geraldo Rogério Faustini Cuzzuol ${ }^{1,2}$ e Andréa Campos Rocha ${ }^{1}$
}

Recebido em 01/03/2011. Aceito em 20/10/2011

\begin{abstract}
RESUMO
(Interação do regime hídrico com as relações nutricionais em ecossistema manguezal). A hipótese de que a sazonalidade do regime fluvial e pluvial tenha alguma influência na composição físico-química do sedimento e na constituição química foliar em arbóreas do ecossistema manguezal foi testada nesse estudo. Foi investigada a influência do regime hídrico na constituição físico-química do ecossistema manguezal do rio Mucuri, Bahia, Brazil. O estudo foi realizado nos meses de abril (estação chuvosa) e outubro (estação de estiagem) nos sítios de domínio de Avicennia germinans, Laguncularia racemosa e Rhizophora mangle. Foram avaliadas as propriedades físico-químicas de seus sedimentos e a análise dos nutrientes das folhas. De maneira geral, os resultados foram influenciados pelo regime hídrico. A época de maior aporte de águas fluviais e pluviais foi marcada pelos maiores valores de bases trocáveis, capacidade de troca catiônica, pH, salinidade e dos nutrientes Ca, Fe e Mn nos sedimentos. Por outro lado, maiores valores de K, Mg e Zn ocorreram no período de estiagem. O regime hídrico influenciou, também, a constituição química das folhas. Durante a estiagem, a concentração de $\mathrm{Ca}, \mathrm{Mg}, \mathrm{Zn}, \mathrm{Fe}$ e $\mathrm{Cu}$ foi maior. Diferenças interespecíficas e variação espacial também foram observadas. As folhas de A. germinans se destacaram pelo acúmulo de nutrientes e $R$. mangle, pela menor concentração de nutrientes. A região do bosque de bacia se caracterizou pelos maiores valores de matéria orgânica, $\mathrm{pH}$ e K. Esses fatores pouco influenciaram na concentração dos nutrientes foliares. A falta de um padrão sazonal claro na geomorfologia, geoquímica e na constituição química das folhas demonstra a complexidade na interpretação de dados em ecossistema costeiro, onde os fatores ambientais interagem de forma sinérgica e antagônica.
\end{abstract}

Palavras-chave: Avicennia germinans, Laguncularia racemosa, sazonalidade, Rhizophora mangle, nutrientes, sedimentos

\begin{abstract}
(Interaction of the water regime with nutrients relations in a mangrove ecosystem). The hypothesis that the seasonality of rainfall and the river regime have some influence on the physical and chemical sediment and chemical composition of tree leaves of the mangrove ecosystem was tested. In this study, the influence of the hydric regime on the physicochemical characteristics of the Mucuri River mangrove ecosystem, in Bahia, Brazil, was evaluated. The study was carried out during the months of April (rainy season) and October (dry season) in sites that were predominantly comprised of Avicennia germinans, Laguncularia racemosa and Rhizophora mangle. We evaluated the physicochemical properties of the sediments and nutrient leaf composition. Overall, the results were influenced by the hydric regime. The period of higher river levels and rainwater intake was marked by high values of exchangeable bases, cation exchange capacity, $\mathrm{pH}$, salinity and $\mathrm{Ca}, \mathrm{Fe}$ and $\mathrm{Mn}$ in the sediments. In contrast, higher values of $\mathrm{K}, \mathrm{Mg}$ and $\mathrm{Zn}$ occurred during the dry season. The hydric regime also influenced the chemical composition of leaves. During the dry season, the concentrations of $\mathrm{Ca}, \mathrm{Mg}, \mathrm{Zn}, \mathrm{Fe}$ and $\mathrm{Cu}$ were higher. Spatial variation and interspecific differences were also observed. Leaves of A. germinans showed a higher accumulation of nutrients, while lower concentration of nutrients were observed in $R$. mangle. The basin forest region (interior of forest) was characterized by high values of organic matter, $\mathrm{pH}$ and $\mathrm{K}$. These factors had little influence on the concentration of nutrients in the leaves. The lack of a clear seasonal pattern in geomorphology, geochemistry and in the chemical composition of the leaves shows the complexity of interpreting data of a coastal ecosystem where environmental factors interact synergistically and antagonistically.
\end{abstract}

Key words: Avicennia germinans, Laguncularia racemosa, nutrients, Rhizophora mangle, seasonality, sediments

1 Universidade Federal do Espírito Santo, Departamento de Ciências Biológicas, Vitória, ES, Brasil

2 Autor correspondente: gcuzzuol@gmail.com 


\section{Introdução}

Reconhecido pela sua contribuição na produção primária de áreas da costa tropical e pelo seu papel na ciclagem de nutrientes, o ecossistema manguezal tem despertado interesse para o estudo da composição química de sua biota em monitoramento ambiental. De maneira geral, resultados têm demonstrado que a composição química das folhas parece refletir as propriedades físico-químicas do ambiente (Lacerda et al. 1988).

Grande parte dos estudos realizados em ecossistema manguezal consiste em levantamentos da composição florística, aspectos estruturais e fitossociológicos, enquanto outros procuraram avaliar a composição química desse ecossistema (Gong \& Ong 1990; Medina et al. 2001; Bernini et al. 2006). Até o momento, poucos trabalhos procuraram correlacionar os fatores ambientais com a composição foliar dos elementos minerais e suas inter-relações em arbóreas do ecossistema manguezal (Cuzzuol \& Campos, 2001; Bernini et al. 2006, Bernini et al. 2010). Uma abordagem mais ampla das interações entre as propriedades físico-químicas dos sedimentos e a composição dos nutrientes foliares em espécies de manguezal pode definir um modelo da dinâmica de ciclagem dos minerais em ecossistemas costeiros.

No sedimento do ecossistema manguezal, os teores de nutrientes variam ao longo da zona entre marés como decorrência das flutuações marinha e do nível de saturação do sedimento que influencia o potencial redox determinante na disponibilidade dos elementos químicos (Tomlinson 1986). No entanto, nem sempre o conteúdo dos nutrientes do sedimento se correlaciona com a concentração dos elementos nas folhas em arbóreas de mangue (Lacerda et al. 1988; Medina et al. 2001). Isso tem permitido inferir que o manguezal possa funcionar como eficiente barreira biogeoquímica dos elementos químicos, especialmente dos metais. Concomitantemente, os mecanismos fisiológicos controladores da absorção de certos elementos químicos podem restringir a absorção de metais pesados (Silva et al. 1990; Lacerda et al. 1995; Lacerda 1997; Machado et al. 2002).

A concentração dos nutrientes minerais em plantas de ecossistemas costeiros pode variar, consideravelmente, em virtude de fatores ambientais, como a freqüência de inundação pelas marés, velocidade de correntes e aporte de água dos rios. A sazonalidade no regime fluvial e pluvial pode interferir na disponibilidade de elementos minerais e alterar a absorção e partição dos elementos minerais em plantas e nas propriedades físico-químicas do substrato (Kjerfve 1990, Bernini et al. 2010).

Partindo desse pressuposto, o objetivo principal deste estudo foi analisar a influência do regime hídrico na composição de elementos minerais em sedimentos e folhas, bem como na constituição físico-química da rizosfera dos bosques onde dominam as arbóreas Avicennia germinans,
Laguncularia racemosa e Rhizophora mangle no estuário do rio Mucuri-BA.

\section{Material e métodos}

\section{Caracterização da área e do ambiente}

O manguezal do rio Mucuri, localizado ao sul do estado da Bahia no município de Mucuri (18 $06^{\circ} 00^{\prime \prime} \mathrm{S}$ e 39³3’30” W), é constituído pelas espécies Avicennia germinans (L.) Stearn., Avicennia schaueriana Staft \& Leechm., Laguncularia racemosa (L.) Gaertn. e Rhizophora mangle L.; além das espécies associadas Acrostichum aureum L. e Hibiscus pernambucensis Arruda. (CEPEMAR 1996). O clima da região é tropical úmido e o estuário está sob o regime micromareal, com marés semidiurnas. No período estudado, a média das marés foi de $0,7 \mathrm{~m}$ (Diretoria de Hidrografia e Navegação, Ministério da Marinha). As espécies estudadas no presente trabalho foram Avicennia germinans (L.) Stearn., Laguncularia racemosa (L.) Gaertn. e Rhizophora mangle L.

Essas três espécies formam agrupamentos ou florestas mistas em determinadas áreas e homogêneos, ou florestas monoespecíficas em outras, destacando-se sempre uma espécie de domínio. Nas áreas homogêneas, Laguncularia racemosa e Rhizophora mangle ocupam a parte inferior do estuário, sob maior influência das marés, e Avicennia germinans restringindo-se a locais próximos à parte superior do estuário (CEPEMAR 1996). Com base nas características fisiográficas desenvolvidas por Lugo \& Snedaker (1974) e reagrupadas por Cintrón et al. (1985), os bosques analisados são do tipo ribeirinho e de bacia, e de formação monoespecífica.

Bosques ribeirinhos são aqueles que se desenvolvem nas margens dos rios e que devido ao fluxo de água rica em nutrientes (descarga de água doce e marés) apresentam elevado porte. Os bosques de bacia se estabelecem nas partes mais internas dos bosques ribeirinhos (Cintrón et al. 1985).

Foram analisadas as médias mensais da temperatura, precipitação e vazão do rio Mucuri de 2004, conforme dados fornecidos pela Estação Meteorológica da Suzano Papel e Celulose-Unidade Mucuri (Fig. 1). A temperatura atmosférica variou apenas $2^{\circ} \mathrm{C}$ entre os meses de abril e outubro, ficando entre 24 e $26^{\circ} \mathrm{C}$. As mais elevadas precipitações ocorreram na estação chuvosa que antecedeu o mês de abril e as mais baixas precipitações, na estação de estiagem, principalmente no mês de outubro. Essa sazonalidade coincidiu com as variações da vazão do rio mais elevada e mais baixa no mês de abril e outubro, respectivamente. Nesses meses de maior amplitude das variações relacionadas ao regime hídrico fluvial, foram realizadas as análises físico-químicas do sedimento e de folhas das árvores do bosque ribeirinho e de bacia de cada estação cuja metodologia é detalhadamente descrita a seguir. 


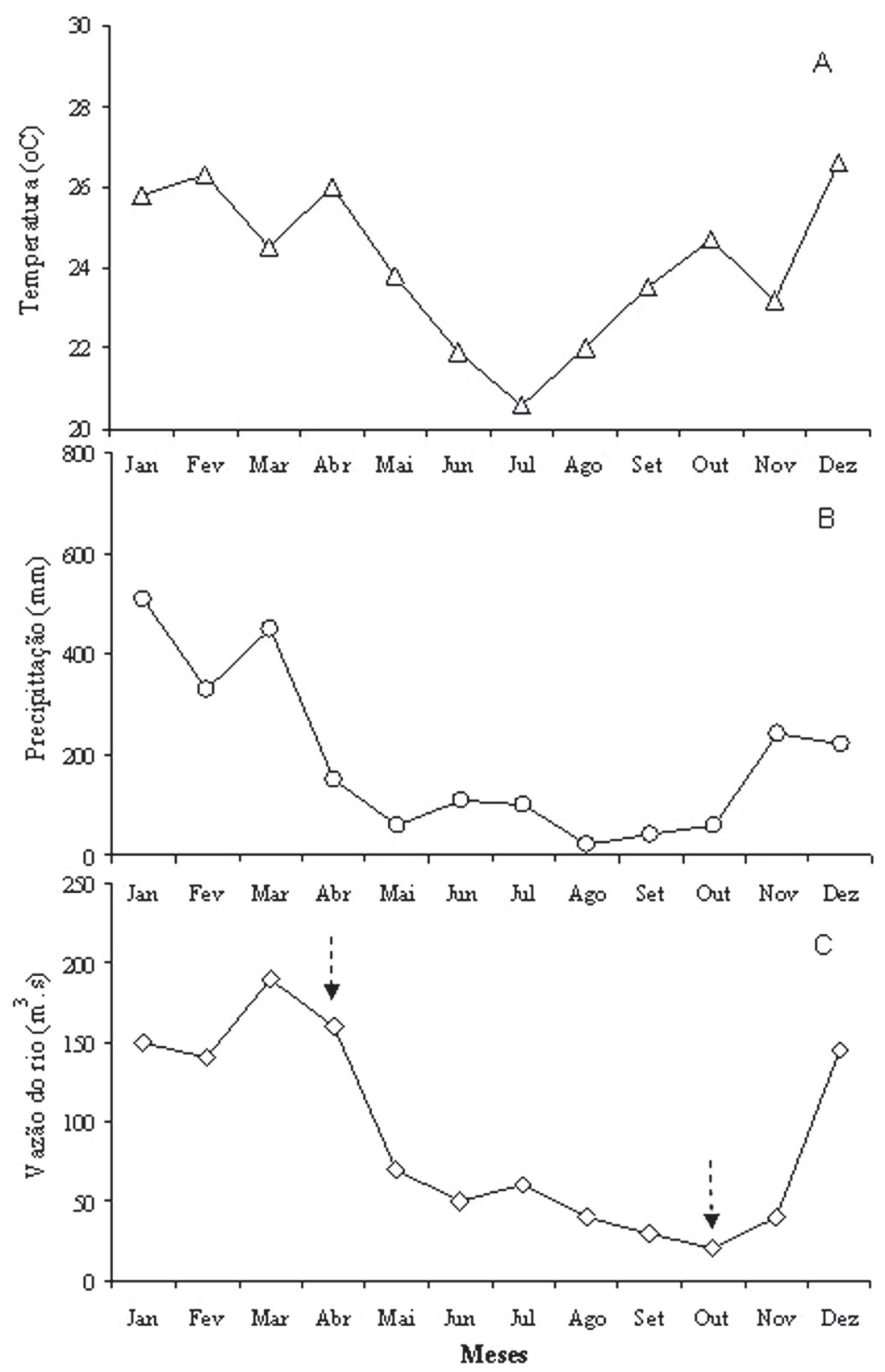

Figura 1. Médias mensais da temperatura (A), precipitação (B) e vazão (C) do rio Mucuri de janeiro a dezembro de 2004. As setas indicam a época de maior e menor vasão do rio do estuário do Rio Mucuri, BA. Fonte: Estação Meteorológica da Suzano Papel e Celulose Unidade Mucuri, 2004.

\section{Análises das características físico-químicas do sedimento e de folhas}

Foram consideradas três estações de estudo, caracterizadas por domínio monoespecíficas, distante entre si num raio de 500-800 metros. Estação 1: Avicennia germinans, Estação 2: Laguncularia racemosa e Estação 3: Rhizophora mangle. Em cada estação foram selecionadas dez árvores, sendo cinco localizadas no bosque ribeirinho e cinco no bosque de bacia, distante aproximadamente $250 \mathrm{~m}$ da margem do rio e então, coletadas amostras do sedimento e de folhas nos meses de abril (maior vazão do rio) e outubro (menor vazão do rio) de 2004, conforme metodologia adotada por Cuzzuol \& Campos (2001). Para cada árvore coletou-se, com sacolas de polietileno, cerca de $0,5 \mathrm{~kg}$ de sedimento da rizosfera (0-10 cm de profundidade) entre as raízes de cada árvore, num raio de $30 \mathrm{~cm}$ do caule, e 50 folhas adultas e verdes do terceiro ou quarto par de folhas dos ramos basais 
marginais expostos ao sol (Cuzzuol \& Campos 2001). As folhas foram acondicionadas em sacolas de polietileno e mantidas em caixas isotérmicas com gelo e, juntamente com as amostras do sedimento, foram conduzidas ao Laboratório de Análises de Solos e de Plantas do Instituto Capixaba de Pesquisa, Assistência Técnica e Extensão Rural (INCAPER) situado no município de Linhares, ES. Assim que as amostras chegaram ao laboratório do INCAPER, as folhas foram lavadas com água destilada e, juntamente com as amostras do sedimento, passaram por um processo de secagem feita em estufa com ventilação forçada sob uma temperatura máxima de $40^{\circ} \mathrm{C}$.

A determinação do $\mathrm{pH}$ da água intersticial do sedimento foi realizada in situ na rizosfera saturada de água de cada árvore (30 $\mathrm{cm}$ de profundidade), utilizando-se $\mathrm{pH}$-metro portátil digital (potenciômetro modelo pHTestr $2^{\mathrm{TM}}$ ). Concomitantemente, mediram-se a salinidade (S\%o) da água intersticial do sedimento ( $30 \mathrm{~cm}$ de profundidade) com refratômetro manual modelo 10049 da American Optical Corporation (USA) e a temperatura $\left({ }^{\circ} \mathrm{C}\right.$ ) do sedimento com termômetro de solo da Hydrolab Surveyor 3. A matéria orgânica (M.O.) do sedimento foi determinada através da oxidação por $\mathrm{Na}_{2} \mathrm{Cr}_{2} \mathrm{O}_{7} 4 \mathrm{Ne} \mathrm{H}_{2} \mathrm{SO}_{4} 10 \mathrm{~N}$. Para a extração de $\mathrm{P}, \mathrm{Na}, \mathrm{K}, \mathrm{Fe}, \mathrm{Zn}, \mathrm{Mn}$ e Cu utilizou-se o extrator Mehlich 1, enquanto o $\mathrm{Ca}, \mathrm{Mg}$ e $\mathrm{Al}$ foram extraídos com o extrator $\mathrm{KCl}$ a $1 \mathrm{M}$. A extração do $\mathrm{B}$ foi realizada em água quente e o $\mathrm{S}$ em solução de fosfato monocálcico em ácido acético. Os métodos utilizados para estas análises são pormenorizadamente descritos por Bataglia et al. (1983) e EMBRAPA (1997).

\section{Análise Estatística}

Os dados foram agrupados por estação (espécie), região (bosque ribeirinho e bacia) e por época de maior e menor vazão do rio. Aplicou-se o teste Kolmogorov-Smirnov para verificação de normalidade e, uma vez confirmada a distribuição normal, os dados foram submetidos à análise de variância (ANOVA). O delineamento experimental foi inteiramente casualizado em esquema fatorial $(3 \times 2 \times 2)$, representando três espécies, duas regiões fisiográficas (regiões ribeirinha e bacia) e duas épocas do ano de vazão do rio: mês de abril, caracterizado pela elevada vazão, e outubro pela baixa vazão. Utilizou-se o programa ASSISTAT versão 7.4 beta (Silva \& Azevedo 2007) e, quando houve significância, aplicou-se o teste de Tukey em 1 e 5\% de nível de significância. Foram consideradas variações espaciais como aquelas ocorridas entre as duas regiões analisadas e variação temporal entre as duas épocas do ano, isto é, abril e outubro.

\section{Resultados e discussão}

\section{Análises físico-químicas do sedimento}

Os sedimentos mostraram-se constituídos, predominantemente, de argila e silte nos sítios de domínio de
Avicennia germinans e Laguncularia racemosa (Tab. 1). Na estação de domínio de Rhizophora mangle, a areia fina foi representativa, com valores equivalentes ao de silte e argila. Valores inferiores dessas duas últimas frações ocorreram em A. germinans e L. racemosa. Resultados semelhantes foram relatados por Cuzzuol \& Campos (2001) no mesmo manguezal de Mucuri, BA confirmando informações anteriores de que as espécies de mangue se estabelecem em substrato argiloso à silte-arenoso (Bernini \& Rezende 2004; Ferreira et al. 2007).

Embora o regime hídrico contribua, efetivamente, na geomorfologia em ecossistemas costeiros (Jímenez 1994, Berrêdo et al. 2008), isso parece não ter ocorrido no manguezal de Mucuri, pois diferença significativa só ocorreu com a fração areia fina, que foi mais representativa na região ribeirinha e em abril, época de maior vazão do rio (Tab. 1). Esse resultado não era esperado uma vez que o maior fluxo das águas (época de maior vazão) proporciona o transporte e depósito de partículas mais densas na região do bosque ribeirinha (Schaeffer-Novelli et al. 1994, Cuzzuol \& Campos 2001). Nesse caso, sugerimos a influência de outro fator ambiental, como a velocidade de vazão do rio, interferindo na deposição das partículas sólida que compõem o sedimento.

Além das variações ambientais como o regime hídrico, outros fatores podem interferir na geomorfologia em ecossistemas manguezais, como o emaranhado de raízes, rizóforos e pneumatóforos, que facilitam a deposição de partículas finas funcionando, dessa forma, como barreiras físicas (Cintrón et al. 1985). As interações significativas encontradas entre as espécies e as regiões de bosque (Tab. 1) são fortes indicativos da influência dos órgãos basais e subterrâneos na dinâmica dos sedimentos do manguezal do rio Mucuri.

Com relação às propriedades físico-químicas dos sedimentos, a estação de domínio de $R$. mangle se destacou pelos maiores teores de M.O. (Tab. 2), confirmando a afirmativa de que espécies do gênero Rhizophora se posicionam em locais de maiores teores de M.O. (Twilley et al. 1995; Cuzzuol \& Campos 2001). Sedimentos de A. germinas e L. racemosa caracterizaram-se pelos maiores valores de $\mathrm{pH}$ do sedimento e da água intersticial (Tab. 2), contrariando informações anteriores de que sítios de maior $\mathrm{pH}$ do manguezal de Mucuri, BA dominam as espécies L. racemosa e R. mangle (Cuzzuol \& Campos 2001). No entanto, essas diferenças nas propriedades físico-químicas do sedimento podem estar relacionadas às variações temporais da maré (Lacerda et al. 1986). Diferente da afirmativa de que espécies do gênero Rhizophora se estabelecem na região inferior do estuário sob maior influência da S\%o (Tomlinson 1986), no presente estudo não foi detectado diferença dessa variável entre as três espécies estudadas. Como discutido anteriormente, variações temporais da maré e na velocidade do rio podem ter interferido nos resultados aqui apresentados.

Os valores de $\mathrm{pH}, \mathrm{V}$ e M.O. parecem ter ligação com a topografia das regiões de bosques, uma vez que maiores va- 
Tabela 1. Valores médios das frações granulométricas do sedimento de Avicennia germinans, Laguncularia racemosa e Rhizophora mangle nos bosques das regiões ribeirinho e de bacia no mês de maior (abril) e menor vazão (outubro) em 2004 no estuário do rio Mucuri, BA. Letras distintas comparam as médias dentro das colunas entre as espécies, as regiões dos bosques e a época do ano em $5 \%$ de significância $(\mathrm{n}=5)$. Os asteriscos $\left(^{*}\right)$ representam $P<0,05$ e $\left(^{(*}\right) P<0,01$.

\begin{tabular}{|c|c|c|c|c|}
\hline \multirow{2}{*}{ Fator de variação } & \multicolumn{4}{|c|}{ Granulometria (\%) } \\
\hline & Areia grossa & Areia fina & Silte & Argila \\
\hline \multicolumn{5}{|c|}{ Espécie vegetal } \\
\hline A. germinans & $5,95 \mathrm{~b}$ & $7,60 \mathrm{c}$ & $36,10 \mathrm{a}$ & $50,30 \mathrm{a}$ \\
\hline L. racemosa & $12,75 \mathrm{a}$ & $15,80 \mathrm{~b}$ & $29,50 \mathrm{~b}$ & $41,95 \mathrm{~b}$ \\
\hline R. mangle & $5,10 \mathrm{~b}$ & $32,65 \mathrm{a}$ & $25,70 \mathrm{c}$ & $34,70 \mathrm{c}$ \\
\hline \multicolumn{5}{|c|}{ Região dos bosques } \\
\hline Ribeirinha & $6,90 \mathrm{a}$ & $20,57 \mathrm{a}$ & $29,97 \mathrm{a}$ & $42,57 \mathrm{a}$ \\
\hline Bacia & $8,97 \mathrm{a}$ & $16,80 \mathrm{~b}$ & $32,23 \mathrm{a}$ & $42,07 \mathrm{a}$ \\
\hline \multicolumn{5}{|c|}{ Época do ano } \\
\hline Abril & $8,50 \mathrm{a}$ & $20,63 \mathrm{a}$ & 29,77 a & $41,10 \mathrm{a}$ \\
\hline Outubro & $7,37 \mathrm{a}$ & $16,73 \mathrm{~b}$ & $32,43 \mathrm{a}$ & $43,53 \mathrm{a}$ \\
\hline \multicolumn{5}{|c|}{ Interação entre os fatores } \\
\hline Estação X Região & ns & ** & $* *$ & ** \\
\hline Estação X Época & ns & ns & ns & ns \\
\hline Região X Época & ns & ns & ns & ns \\
\hline
\end{tabular}

lores dessas variáveis foram encontrados na região de bacia (Tab. 2). O acúmulo de M.O. na região do bosque de bacia sugere que seus sedimentos sejam retentores de M.O. (Jorcin 2000), em virtude da baixa energia em sítios onde se formam depressões no ecossistema manguezal (Siqueira et al. 2006).

O maior pH no mês de outubro em relação ao mês de abril (Tab. 2) foi associado à menor precipitação e vazão do rio (Fig. 1). Isso sugere que os maiores valores dos componentes relacionados às bases trocáveis (SB, CTC, V) e da S\%o em outubro (Tab. 2) podem ser apontados como possíveis fatores de variação do $\mathrm{pH}$ entre as épocas do ano. Dessa forma, a sazonalidade do regime hídrico parece se relacionar com o pH dos sedimentos devido à sua grande influência nas propriedades geoquímicas (Berrêdo et al. 2008).

De todas as variáveis relacionadas às propriedades físico-químicas, a $\mathrm{S} \%$ o foi a que mostrou maior amplitude de variação (Tab. 2). Sua concentração foi, expressivamente, superior na época do ano de menor vazão do rio. A relação entre $S \%$ e o regime hídrico é bem conhecida em ecossistemas manguezais (Cohen et al. 1999; Berrêdo et al. 2008; Bernini et al. 2010), em que a maior salinidade da água intersticial dos sedimentos ocorre na estação de estiagem como constatado no presente estudo. A temperatura dos sedimentos das três espécies vegetais estudadas, também, apresentou variação sazonal (Tab. 2). A maior temperatura na época de maior vazão (abril) pode ser associada às elevadas temperaturas atmosféricas na estação mais chuvosa (Fig. 1). Além das variações sazonais do clima, fatores bióticos como a contribuição da estrutura da vegetação no aumento da temperatura do sedimento em períodos de maior precipitação tem sido sugerida por Souza et al. (2004). Isso se deve à maior atividade metabólica das células radiculares em resposta ao aumento da temperatura do solo (Marschner 1995).

A relação das propriedades físico-químicas do sedimento com o regime hídrico pode ser confirmada mais uma vez, através da análise da interação das espécies e regiões dos bosques com a época do ano (Tab. 2). Nota-se que a interação entre as espécies e tipo de região do bosque foi significativa para todas as variáveis, exceto para a S\%o. Quanto a interação espécies/região do bosque com a época do ano, diferenças significativas ocorreram para M.O., $\mathrm{pH}$ e $\mathrm{T}^{\mathrm{b}} \mathrm{C}$ demonstrando a grande influência da sazonalidade do regime hídrico na dinâmica dos sedimentos em ecossistemas costeiro de clima equatorial (Fiot \& Gratiot 2006). Corroborando com essas informações, Berrêdo et al. (2008) concluíram que a sazonalidade anual das chuvas tem grande impacto nas propriedades sedimentológicas dos manguezais da região amazônica. Nesse aspecto, o regime hídrico modificou as propriedades físico-químicas dos sedimentos no manguezal do rio Mucuri (Tab. 2). De maneira geral, podemos concluir que a porcentagem de areia fina e $\mathrm{a} \mathrm{T}^{\mathrm{b}} \mathrm{C}$ foi maior na época de chuvas, enquanto os componentes relacionados às bases trocáveis (SB, CTC e V) e a salinidade foram maiores na estação de estiagem.

\section{Macro e micronutrientes dos sedimentos}

Sedimentos de A germinans e L. racemosa apresentaram maiores teores de $\mathrm{P}, \mathrm{K} \mathrm{e} \mathrm{Cu}$ (Tab. 3). Já os sedimentos de $R$. mangle se destacaram pelos maiores valores de $\mathrm{Ca}, \mathrm{Fe}$ e Zn, confirmando os resultados levantados por Cuzzuol \& Campos (2001) no mesmo manguezal. Diferente dos resultados desses autores que relataram maiores valores 
Tabela 2. Valores médios das propriedades físico-químicas do sedimento de Avicennia germinans, Laguncularia racemosa e Rhizophora mangle nos bosques das regiões ribeirinho e de bacia, nos meses de maior vazão (abril) e menor vazão (outubro) de 2004 no Manguezal do estuário do rio Mucuri, BA. M.O.= matéria orgânica,

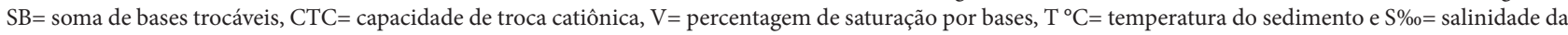
água intersticial. Letras distintas comparam as médias dentro das colunas entre as espécies, as regiões dos bosques e a época do ano em $5 \%$ de significância ( $\mathrm{n}=5$ ). Os asteriscos $\left(^{*}\right)$ representam $P<0,05$ e $\left.{ }^{(*}\right) P<0,01$.

\begin{tabular}{|c|c|c|c|c|c|c|c|}
\hline Fator de Variação & $\begin{array}{c}\text { M.O. } \\
\left(\mathrm{g} . \mathrm{Kg}^{-1}\right)\end{array}$ & $\begin{array}{c}\mathrm{SB} \\
\left(\mathrm{cmol}_{\mathrm{c}} \cdot \mathrm{dm}^{-3}\right)\end{array}$ & $\begin{array}{c}\text { CTC } \\
\left(\mathrm{mmol}_{\mathrm{c}} \cdot \mathrm{d}^{-3}\right)\end{array}$ & $\begin{array}{c}\mathrm{V} \\
(\%)\end{array}$ & $\mathrm{pH}$ & $\mathrm{T}\left({ }^{\circ} \mathrm{C}\right)$ & S (\%o) \\
\hline \multicolumn{8}{|c|}{ Espécie Vegetal } \\
\hline A. germinans & $33 \mathrm{~b}$ & $21 \mathrm{~b}$ & $237 \mathrm{~b}$ & $88 \mathrm{~b}$ & $6,7 \mathrm{~b}$ & $26 \mathrm{a}$ & $11,3 \mathrm{~b}$ \\
\hline L. racemosa & $33 \mathrm{~b}$ & $21 \mathrm{~b}$ & $247 \mathrm{~b}$ & $85 \mathrm{~b}$ & $6,9 \mathrm{~b}$ & $27 \mathrm{a}$ & $11,2 \mathrm{~b}$ \\
\hline R. mangle & $40 \mathrm{a}$ & $25 \mathrm{a}$ & $277 \mathrm{a}$ & $90 \mathrm{a}$ & $7,7 \mathrm{a}$ & $27 \mathrm{a}$ & $19,5 \mathrm{a}$ \\
\hline \multicolumn{8}{|c|}{ Região dos bosques } \\
\hline Ribeirinha & $30 \mathrm{~b}$ & $22 \mathrm{a}$ & $251 \mathrm{a}$ & $87 \mathrm{a}$ & $6,6 \mathrm{~b}$ & $26 \mathrm{a}$ & $10,5 \mathrm{a}$ \\
\hline Bacia & $40 \mathrm{a}$ & $23 \mathrm{a}$ & $256 \mathrm{a}$ & $89 a$ & $7,6 \mathrm{a}$ & $27 \mathrm{a}$ & $10,8 \mathrm{a}$ \\
\hline \multicolumn{8}{|c|}{ Época do ano } \\
\hline Abril & $33 \mathrm{a}$ & $19 \mathrm{~b}$ & $230 \mathrm{~b}$ & $82 \mathrm{~b}$ & $6,5 \mathrm{~b}$ & $28 \mathrm{a}$ & $09,2 \mathrm{~b}$ \\
\hline Outubro & $37 \mathrm{a}$ & $25 \mathrm{a}$ & $284 \mathrm{a}$ & $88 \mathrm{a}$ & $7,4 \mathrm{a}$ & $25 \mathrm{~b}$ & $15,2 \mathrm{a}$ \\
\hline \multicolumn{8}{|c|}{ Interação entre os fatores } \\
\hline Espécie X Região & $* *$ & ** & $* *$ & $* *$ & ** & $* *$ & ns \\
\hline Espécie X Época & * & ns & ns & ns & ** & $* *$ & ns \\
\hline Região X Época & ns & ns & ns & * & ** & ** & ns \\
\hline
\end{tabular}

Mg em sedimentos de L. racemosa e R. mangle e, de Mn no sedimento de L. racemosa, no presente trabalho não foram encontradas diferenças desses elemento entre as três espécies. Este resultado pode ser atribuído, mais uma vez, à variação temporal em relação ao trabalho de Cuzzuol \& Campos (2001) e à instabilidade sazonal dos sedimentos em ecossistemas costeiros (Bernini et al. 2010) em função das flutuações da maré, que tem grande efeito na disponibilidade dos elementos químicos (Lacerda et al. 1986). Um outro fator a ser considerado é o espacial, o qual tem grande efeito nas propriedades físico-químicas dos sedimentos (Bernini et al. 2006, 2010). As estações do presente estudo foram deslocadas para a região mais superior do manguezal, devido às alterações da vegetação constatada em relação ao trabalho de Cuzzuol \& Campos (2001).

A topografia do relevo das regiões dos bosques influenciou na concentração de $\mathrm{K}, \mathrm{Fe}$ e $\mathrm{Cu}$. Se por um lado a região de bacia mostrou-se mais rica em $\mathrm{K}$, o bosque da região ribeirinha se destacou pelas maiores concentrações de $\mathrm{Fe}$ e $\mathrm{Cu}$ (Tab. 3). Esses resultados confirmam informações anteriores de que o bosque de bacia tende acumular maiores concentrações de $\mathrm{K}$ em relação ao bosque ribeirinho, especialmente, nos sítios de domínio de $L$. racemosa (Cuzzuol \& Campos 2001). Diferente do trabalho atual, esses autores não encontraram nenhuma diferença na concentração do $\mathrm{Fe}$, mas sim para o $\mathrm{Cu}$, que se acumulou na região de bacia onde a deposição dos elementos químicos tende a ser expressiva. As diferenças encontradas no presente estudo em relação ao estudo anterior no mesmo manguezal do rio Mucuri, BA (Cuzzuol \& Campos 2001) e em comparação aos manguezais de outras localidades sob condições ambientais semelhantes, como o manguezal do rio São Mateus, ES (Bernini et al. 2006), demonstram que as propriedades físico-químicas do sedimento do manguezal estão sob grande influência da variação temporal e espacial (Bernini et al. 2010).

Além das diferenças interespecíficas e da influência espacial, o regime hídrico teve grande efeito na composição dos macro e micronutrientes dos sedimentos (Tab. 3). A maior precipitação e a vazão do rio em abril foram associadas ao acúmulo de $\mathrm{Ca}, \mathrm{Fe}$ e $\mathrm{Mn}$, enquanto os maiores valores de $\mathrm{K}, \mathrm{Mg}$ e $\mathrm{Zn}$ ocorreram em outubro, caracterizado pela menor precipitação e vazão do rio (Tab. 3). O acúmulo catiônico ( $\mathrm{K} \mathrm{e} \mathrm{Mg)} \mathrm{no} \mathrm{período} \mathrm{de} \mathrm{estiagem}$ assinala a intrusão de águas oceânicas no estuário conforme sugerido por Cuzzuol \& Campos (2001) e Berrêdo et al. (2008). O acúmulo do Ca na época de maior precipitação e vazão do rio não era esperado, uma vez que as bases trocáveis são fornecidas, em maior proporção, pelas águas oceânicas (Cohen et al. 1999). O maior teor de Ca, também, encontrado na estação chuvosa no manguezal de São Mateus, ES, permitiu que Bernini et al. (2010) justificassem a dificuldade de interpretação dos resultados, devido à falta de um padrão sazonal na concentração de todos os nutrientes. Isso nos leva a supor que outros fatores ambientais não avaliados no presente trabalho, como precipitações repentinas nos dias anteriores à coleta de dados, tenham interferido nos resultados.

Interações significativas entre as espécies e regiões dos bosques ocorreram para todos os elementos químicos 
Tabela 3. Valores médios em mg.dm³ de macro e micronutrientes do sedimento de Avicennia germinans, Laguncularia racemosa e Rhizophora mangle nos bosques das regiões ribeirinho e de bacia, nos meses de maior vazão (abril) e menor vazão (outubro) de 2004 no Manguezal do estuário do rio Mucuri, BA. Letras distintas comparam as médias dentro das colunas entre as espécies, as regiões dos bosques e a época do ano em $5 \%$ de significância $(\mathrm{n}=5)$. Os asteriscos $\left({ }^{\star}\right)$ representam $P$ $<0,05$ e $\left.{ }^{* *}\right) P<0,01$.

\begin{tabular}{|c|c|c|c|c|c|c|c|c|}
\hline Fator de variação & $\mathrm{P}$ & $\mathrm{K}$ & $\mathrm{Ca}$ & $\mathrm{Mg}$ & $\mathrm{Fe}$ & $\mathrm{Cu}$ & $\mathrm{Mn}$ & $\mathrm{Zn}$ \\
\hline \multicolumn{9}{|c|}{ Espécie vegetal } \\
\hline A. germinans & $16,1 \mathrm{a}$ & $448 \mathrm{a}$ & $746 \mathrm{~b}$ & $847 \mathrm{a}$ & $1.976 \mathrm{~b}$ & $1,87 \mathrm{a}$ & $75 \mathrm{a}$ & $9,06 \mathrm{a}$ \\
\hline L. racemosa & $15,3 \mathrm{a}$ & $406 \mathrm{a}$ & $808 \mathrm{~b}$ & $843 \mathrm{a}$ & $1.749 \mathrm{c}$ & $1,45 \mathrm{~b}$ & $62 \mathrm{a}$ & $9,58 \mathrm{a}$ \\
\hline R. mangle & $13,4 \mathrm{~b}$ & $349 \mathrm{~b}$ & $1.072 \mathrm{a}$ & $960 \mathrm{a}$ & $2.335 \mathrm{a}$ & $1,05 \mathrm{c}$ & $63 \mathrm{a}$ & $11,31 \mathrm{a}$ \\
\hline \multicolumn{9}{|c|}{ Região do bosque } \\
\hline Ribeirinha & $14,5 \mathrm{a}$ & $376 \mathrm{~b}$ & $840 \mathrm{a}$ & $847 a$ & $2.109 \mathrm{a}$ & $1,51 \mathrm{a}$ & $73 a$ & $9,42 \mathrm{a}$ \\
\hline Bacia & $15,3 \mathrm{a}$ & $426 \mathrm{a}$ & $910 \mathrm{a}$ & $920 \mathrm{a}$ & $1.931 \mathrm{~b}$ & $1,40 \mathrm{~b}$ & $61 \mathrm{a}$ & $10,56 \mathrm{a}$ \\
\hline \multicolumn{9}{|c|}{ Época do ano } \\
\hline Abril & $14,9 \mathrm{a}$ & $299 \mathrm{~b}$ & $1.026 \mathrm{a}$ & $542 \mathrm{~b}$ & $2.401 \mathrm{a}$ & $1,38 \mathrm{a}$ & $90 \mathrm{a}$ & $8,57 \mathrm{~b}$ \\
\hline Outubro & $15,0 \mathrm{a}$ & $502 \mathrm{a}$ & $722 \mathrm{~b}$ & $1.224 \mathrm{a}$ & $1.639 \mathrm{~b}$ & $1,54 \mathrm{a}$ & $43 \mathrm{~b}$ & $11,40 \mathrm{a}$ \\
\hline \multicolumn{9}{|c|}{ Interação entre os fatores } \\
\hline Espécie X Região & $* *$ & $* *$ & $* *$ & $* *$ & $* *$ & $* *$ & $* *$ & $* *$ \\
\hline Espécie X Época & * & * & * & ns & ns & ** & ns & ns \\
\hline Região X Época & ns & $* *$ & ns & ns & ns & * & ns & ns \\
\hline
\end{tabular}

(Tab. 3). Interações entre espécies e épocas do ano só foram significativas para $\mathrm{P}, \mathrm{K}, \mathrm{Ca}$ e $\mathrm{Cu}$, enquanto interações significativas entre regiões dos bosques e época do ano só foram observadas para $\mathrm{Ke} \mathrm{Cu}$. Esses resultados confirmam indicações anteriores de que a época do ano de menor vazão do rio Mucuri favoreceu o acúmulo de $\mathrm{K} \mathrm{e} \mathrm{Mg,}$ envolvendo a participação das águas oceânicas, grande contribuinte na disponibilização dessas bases trocáveis (Cohen et al. 1999).

\section{Macro e micronutrientes foliares}

De maneira geral, as folhas de A. germinans mostraram-se mais ricas em nutrientes, seguido por $L$. racemosa e R. mangle (Tab. 4), assim como reportado anteriormente (Lacerda et al. 1986, Cuzzuol \& Campos 2001, Bernini et al. 2006). Nesse aspecto, A. germinans se destacou pelas maiores concentrações de $\mathrm{N}, \mathrm{K}, \mathrm{Mg}$ e Zn, enquanto $L$. racemosa chamou a atenção pelos maiores teores de $\mathrm{P}$ e $\mathrm{Cu}$ e pela elevadíssima concentração de Fe (Tab. 4). Outra informação interessante quanto aos nutrientes foliares está na similaridade entre $L$. racemosa e $R$. mangle, que apresentaram os maiores valores de $\mathrm{Ca}$ e menores de $\mathrm{N}$.

Em parte, as diferenças interespecíficas se devem às propriedades físico-químicas dos sedimentos. De acordo com Lacerda et al. (1988) e Twilley et al. (1995), os sedimentos ocupados por $L$. racemosa e $R$. mangle são capazes de acumular e imobilizar os macronutrientes, principalmente $o$ $\mathrm{N}$, afetando diretamente a fertilidade do solo.

Além da geoquímica, as exigências nutricionais interespecíficas das três arbóreas investigadas são influenciadas, também, pelas relações sinérgicas e antagônicas na absorção dos macro e micronutrientes. Waisel (1972) afirma que a absorção do elemento Ca é controlada de forma antagônica pelo nível de $\mathrm{Na}$ acumulado no sedimento. No entanto, na época de maior vazão do rio Mucuri, caracterizada pela menor S\%o, a concentração foliar do Ca foi menor (Tab. 2 e 4). Isso pode ter ocorrido em função dos maiores valores de SB e pelo caráter ácido dos sedimentos na época de menor fluxo das águas fluviais (Tab. 2). Embora o Ca tenha-se acumulado nos sedimentos na época de maior vazão do rio (Tab. 3), a absorção do Ca não foi estimulada pela maior disponibilidade desse elemento no mês de abril mostrando que outros fatores como as exigências nutricionais interespecíficas e as propriedades físico-químicas dos sedimentos estejam envolvidas.

Além do Ca, a $\mathrm{S} \%$, também, tem relação com a absorção de micronutrientes em arbóreas de ecossistema manguezal (Lacerda et al. 1988). O fato de $R$. mangle apresentar um mecanismo de exclusão de sal na raiz (Tomlinson 1986) poderia limitar a entrada de metais como $\mathrm{Zn}$, Fe e Cu (Cuzzuol \& Campos 2001) como constatado no presente trabalho (Tab. 4). Por outro lado, $R$. mangle não dispõe de mecanismos para limitar a absorção do $\mathrm{Mn}$ ou de eliminá-lo no nível radicular ou foliar (Tomlinson 1986) o que deve ter contribuído para o maior acúmulo foliar de Mn em R. mangle (Tab. 4). Já em L. racemosa, por apresentar um mecanismo do tipo sal-includente, possui elevada capacidade de limitar a absorção e translocação do $\mathrm{Mn}$, mas não possui mecanismo para impedir o acúmulo de Fe (Tomlinson 1986) conforme verificado no presente trabalho (Tab. 4).

Os elevados valores de Mn em folhas de R. mangle e, de Fe em L. racemosa (Tab. 4), também já haviam sido relatados anteriormente no manguezal de Mucuri, BA (Cuzzuol 
Tabela 4. Valores médios das concentrações de macro e micronutrientes foliares nas espécies Avicennia germinans, Laguncularia racemosa e Rhizophora mangle nos bosques das regiões ribeirinho e de bacia, nos meses de maior vazão (abril) e menor vazão (outubro) de 2004 no Manguezal do estuário do rio Mucuri, BA. Letras distintas comparam as médias dentro das colunas entre as espécies, as regiões dos bosques e a época do ano em $5 \%$ de significância $(\mathrm{n}=5)$. Os asteriscos $\left({ }^{\star}\right)$ representam $P<0,05$ e $\left.{ }^{* *}\right) P<0,01$.

\begin{tabular}{|c|c|c|c|c|c|c|c|c|c|c|c|}
\hline Fator de & $\mathrm{N}$ & $\mathrm{P}$ & K & $\mathrm{Ca}$ & $\mathrm{Mg}$ & S & $\mathrm{Zn}$ & $\mathrm{Fe}$ & $\mathrm{Mn}$ & $\mathrm{Cu}$ & B \\
\hline variação & \multicolumn{6}{|c|}{ g. $\mathrm{kg}^{-1}$} & \multicolumn{5}{|c|}{ mg.kg-1 } \\
\hline \multicolumn{12}{|c|}{ Espécies vegetais } \\
\hline A. germinans & $20,0 \mathrm{a}$ & $1,3 \mathrm{~b}$ & $12,0 \mathrm{a}$ & $0,68 \mathrm{~b}$ & $0,73 \mathrm{a}$ & $2,2 \mathrm{a}$ & $20,7 \mathrm{a}$ & $180 \mathrm{~b}$ & $201 \mathrm{~b}$ & $1,06 \mathrm{~b}$ & $26,3 \mathrm{c}$ \\
\hline L. racemosa & $14,4 \mathrm{~b}$ & $1,6 \mathrm{a}$ & $9,9 \mathrm{~b}$ & $1,84 \mathrm{a}$ & $0,48 \mathrm{c}$ & $2,3 \mathrm{a}$ & $20,7 \mathrm{a}$ & $1.557 \mathrm{a}$ & $095 \mathrm{c}$ & $1,71 \mathrm{a}$ & $33,4 \mathrm{~b}$ \\
\hline R. mangle & $14,8 \mathrm{~b}$ & $1,1 \mathrm{c}$ & $9,0 \mathrm{~b}$ & $1,65 \mathrm{a}$ & $0,59 \mathrm{~b}$ & $2,1 \mathrm{a}$ & $6,5 \mathrm{~b}$ & $56 \mathrm{c}$ & $594 \mathrm{a}$ & $0,14 \mathrm{c}$ & $56,0 \mathrm{a}$ \\
\hline \multicolumn{12}{|c|}{ Região do bosque } \\
\hline Ribeirinha & $15,5 \mathrm{~b}$ & $1,3 \mathrm{a}$ & $11,2 \mathrm{a}$ & $1,39 \mathrm{a}$ & $0,59 \mathrm{a}$ & $2,2 \mathrm{a}$ & $16,6 \mathrm{a}$ & $554 \mathrm{a}$ & $313 \mathrm{a}$ & $1,10 \mathrm{a}$ & $40,2 \mathrm{a}$ \\
\hline Bacia & $17,2 \mathrm{a}$ & $1,4 \mathrm{a}$ & $9,4 \mathrm{~b}$ & $1,39 \mathrm{a}$ & $0,61 \mathrm{a}$ & $2,2 \mathrm{a}$ & $15,3 \mathrm{a}$ & $641 \mathrm{a}$ & $281 \mathrm{a}$ & $0,84 \mathrm{a}$ & $36,9 \mathrm{~b}$ \\
\hline \multicolumn{12}{|c|}{ Época do ano } \\
\hline Abril & $16,3 \mathrm{a}$ & $1,3 \mathrm{a}$ & $10,1 \mathrm{a}$ & $0,76 \mathrm{~b}$ & $0,39 \mathrm{~b}$ & $2,2 \mathrm{a}$ & $14,6 \mathrm{~b}$ & $514 \mathrm{~b}$ & $267 \mathrm{a}$ & $0,26 \mathrm{~b}$ & $40,4 \mathrm{a}$ \\
\hline Outubro & $16,5 \mathrm{a}$ & $1,4 \mathrm{a}$ & $10,5 \mathrm{a}$ & $2,02 \mathrm{a}$ & $0,81 \mathrm{a}$ & $2,2 \mathrm{a}$ & $17,3 \mathrm{a}$ & $681 \mathrm{a}$ & $326 \mathrm{a}$ & $1,68 \mathrm{a}$ & $36,6 \mathrm{a}$ \\
\hline \multicolumn{12}{|c|}{ Interação entre os fatores } \\
\hline Espécie X Região & ns & ns & * & ns & ns & ns & ns & ns & ns & $* *$ & ns \\
\hline Espécie X Época & $* *$ & $* *$ & $* *$ & $* *$ & $* *$ & ** & $* *$ & $* *$ & ns & $* *$ & $* *$ \\
\hline Região X Época & ns & ns & ns & ns & ns & ns & ns & ns & ns & $* *$ & ns \\
\hline
\end{tabular}

\& Campos 2001) e de São Mateus, ES (Bernini et al. 2006). Essa particularidade permitiu Lacerda et al. (1988) inferirem que o substrato onde se desenvolvem estas duas espécies possui elevada capacidade de retenção de Mn e Fe e, por isso, encontram-se mais adaptadas em povoar ambientes ricos em Mn e Fe (Twilley et al. 1995). Dessa forma, o sedimento ocupado por L. racemosa e R. mangle possui elevada capacidade em acumular e imobilizar os macronutrientes, refletindo na baixa concentração foliar do $\mathrm{N}$ e do $\mathrm{K}$ (Tab. 4), mesmo com $R$. mangle se estabelecendo em sedimentos com maiores valores de M.O e CTC (Tab. 2). Esses resultados sugerem que arbóreas de manguezal sejam capazes de expressar sua seletividade na absorção dos elementos químicos independente da maior ou menor disponibilidade dos nutrientes no substrato.

Quanto à influência espacial, poucas foram as diferenças encontradas nos teores de nutrientes foliares entre as regiões ribeirinha e de bacia. Nesse aspecto, o bosque da região ribeirinha se caracterizou pelos maiores teores de $\mathrm{K}$ e Fe enquanto o bosque de bacia, pelos maiores valores de $\mathrm{N}$ (Tab. 4). Esses resultados demonstram que o fator espacial não teve grande influência na constituição química foliar das espécies estudadas, embora haja citações de que as propriedades físico-químicas dos sedimentos diferem entre esses dois tipos de bosques (Bernini et al. 2006, 2010).

Como constatado para nas análises dos sedimentos (Tab. 1, 2 e 3), o regime hídrico teve grande influência na concentração dos nutrientes foliares (Tab. 4). No mês de outubro, marcado pela menor precipitação e vazão do rio, as folhas acumularam maiores teores de $\mathrm{Ca}, \mathrm{Mg}, \mathrm{Zn}, \mathrm{Fe}$ e Cu. Esses resultados demonstram, mais uma vez, o grande impacto do regime hídrico na absorção iônica de alguns elementos como constatado pela interação entre espécie vegetal e época do ano para a maioria dos elementos analisados (Tab. 4). Dessa forma, períodos de estiagem podem elevar a S\%o, SB e CTC (Tab. 2) influenciando, de forma sinérgica, a absorção radicular (Lara \& Dittmar 1999), culminando no acúmulo de nutrientes minerais no mês de outubro (Tab. 4). Tal variação temporal no conteúdo de nutrientes em folhas de plantas adultas de manguezal, também, foi relatada por Wang et al. (2003). Porém, nem sempre se consegue encontrar um padrão sazonal claro na concentração de nutrientes minerais (Bernini et al. 2010), demonstrando a complexidade na interpretação de dados em um ambiente onde os fatores interagem de forma sinérgica e antagônica (Cuzzuol \& Campos 2001).

De qualquer forma, os resultados apresentados no presente estudo indicam que as concentrações dos nutrientes dos sedimentos e das folhas são suscetíveis à influência do regime hídrico. Da mesma forma, o $\mathrm{pH}$ do sedimento, a $\mathrm{T}^{\mathrm{b}} \mathrm{C}$, a $S \%$ e a SB, também, foram influenciados pelas variações da precipitação e do fluxo do rio. Independente da concentração dos nutrientes minerais nos sedimentos, as espécies mostraram proporções interespecíficas de concentração dos minerais que foram, em grande parte, maiores na época de menor vazão do rio (outubro). Dessa maneira, além do fator espacial (região do bosque), as propriedades físico-químicas dos sedimentos e a absorção iônica interespecífica de A. germinans, L. racemosa e $R$. mangle estão sujeitos às variações climáticas como a precipitação e a hidrodinâmica do rio do manguezal do rio Mucuri, BA. 


\section{Agradecimentos}

Ao Centro de Pesquisas do Mar pelo apoio logístico.

\section{Referências bibliográficas}

Bataglia, O.C.; Furlani, A.M.C.; Teixieira, J.P.F.; Furlani, P.R. \& Gallo, J.R. 1983. Métodos de análise química de plantas. Boletim Técnico 78. Instituto Agronômico de Campinas.

Bernini E. \& Rezende, C.E. 2004. Estrutura da vegetação em florestas de mangue do estuário do rio Paraíba do Sul, Estado do Rio de Janeiro, Brasil. Acta Botanica Brasilica 18(3): 491-502.

Bernini, E.; Silva, M.A.B.; Carmo, T.M.S. \& Cuzzuol, G.R.F. 2006. Composição química do sedimento e de folhas das espécies do manguezal do estuário do rio São Mateus, Espírito Santo, Brasil. Revista Brasileira de Botânica 29(4): 689-699.

Bernini, E.; Da Silva, M.A.B.; Do Carmo, T.M.S. \& Cuzzuol, G.R.F. 2010. Spatial and temporal variation of the nutrients in the sediment and leaves of two Brazilian mangrove species and their role in the retention of environmental heavy metals. Brazilian Journal of Plant Physiology 22(3): 177-187.

Berrêdo, J.F.; Costa, M.L. \& Progene, M.P.S. 2008. Efeitos das variações sazonais do clima tropical úmido sobre as águas e sedimentos de manguezais do estuário do rio Marapanim, costa nordeste do Estado do Pará. Acta Amazonica 38(3): 473-482.

CEPEMAR. Centro de Pesquisas do Mar. 1996. Monitoramento do manguezal do rio Mucuri-BA. Relatório Técnico Final - RTF 067/96, v.2.

Cintrón, G.; Lugo, A.E. \& Martínez, R. 1985. Structural and functional properties of mangrove forests. Pp.53-66. In Dárcy, W.G. \& Correa, M.D. (Orgs.). The Botany and Natural History of Panama. Saint Louis, Missouri Botanical Garden.

Cohen, M.C.L.; Lara, R.J.; Ramos, J.F. \& Dittmar, T. 1999. Factors influencing the variability of magnesium, calcium and potassium in waters of a mangrove creek in Bragança, North Brazil. Mangroves and Salt Marshes 3: 9-15.

Cuzzuol, G.R.F. \& Campos, A. 2001. Aspectos nutricionais na vegetação de manguezal do estuário do rio Mucuri, Bahia, Brasil. Revista Brasileira de Botânica 24: 227-234.

EMBRAPA. Empresa Brasileira de Pesquisa Agropecuária. 1997. Centro Nacional de Pesquisas de Solos. Manual de Métodos de Análises de Solo. Rio de Janeiro, EMBRAPA-CNPS.

Fiot, J. \& Gratiot, N. 2006. Structural effects of tidal exposures on mudflats along the Frehc Guiana coast. Marine Geology 228: 25-37.

Ferreira, T.O.; Otero, X.L.; Vidal-Torrado, P. \& Macías, F. 2007. Effects of bioturbation by root and crab activity on iron and sulfur biogeochemistry in mangrove substrate. Geoderma 142(1): 36-46.

Gong, W.K. \& Ong, J.E. 1990. Plant biomass and nutrient flux in a managed mangrove forest in Malaysia. Estuarine, Coastal and Shelf Science 31(5): 516-530.

Jímenez, J.A. 1994. Los manglares del Pacifico de Centroamérica. Heredia, Editorial Fundación UMA.

Jorcin, A. 2000. Physical and chemical characteristics of the sediment in the estuarine region of Cananéia (SP), Brazil. Hydrobiologia 431(1): 59-67.

Kjerfve, B. 1990. Manual for investigation of hydrological processes in mangrove ecosystems. New Delhi, UNESCO/UNDP.

Lacerda, L.D.; Rezende, C.E.; José, D.M.V. \& Francisco, M.C.F. 1986. Metallic compositon of mangrove leaves from the southeastern Brazilian coast. Revista Brasileira de Biologia 46(1): 395-399.
Lacerda, L.D.; Martinelli, L.A.; Rezende, C.E.; Mozeto, A.A.; Ovalle, A.R.C.; Victoria, R.L.; Silva, C.A.R. \& Nogueira, F.B. 1988. The fate of trace metals in suspended matter in a mangrove creek during a tidal cycle. The Science of the Total Environmental 75(2): 169-180.

Lacerda, L.D.; Ittellpt, V. \& Patchineelam, S.R. 1995. Biochemistry of mangrove soil organic matter: a comparison between Rhizophora and Avicennia soils in south-eastern Brazil. Estuarine, Coastal and Shelf Science 40: 713-720.

Lacerda, L.D. 1997. Trace metals in mangrove plants: why such low concentrations? Pp. 171-178. In Kjerfve, B.; Lacerda, L.D. \& Diop, H.S. (Orgs.). Mangrove Ecosystem Studies in Latin America and Africa. Paris, Unesco.

Lara, R.J. \& Dittmar, T. 1999. Nutrient dynamics in a mangrove creek (North Brazil) during the dry season. Mangroves and Salt Marshes 3(3): 185-195

Lugo, A.E. \& Snedaker, S.C. 1974. The ecology of mangroves. Annual Review of Ecology and Systematic 5(1): 39-64.

Machado, W.; Moscatelli, M.; Rezende, L.G. \& Lacerda, L.D. 2002. Mercury, zinc and copper accumulation in mangrove sediments surrounding a large landfill in southeast Brazil. Environmental Pollution 120(2): 455-461.

Marschner, H. 1995. Mineral nutrition of higher plants. $2^{\text {nd }}$ ed. Orlando, Academic Press.

Medina, E.; Glarizzo, T.; Menezes, M.; Carvalho, L.M.; Carvalho, E.A.; Peres, A.; Silva, B.; Vilhena, R.R.A. \& Braga, F.C. 2001. Mangal communities of the Salgado Paraense: ecological heterogenecity alone the Bragança península assessed through soil and leaf analysis. Amazoniana 16(4): 397-416.

Schaeffer-Novelli, Y.; Peria, L.C.S.; Menezes, G.V.; Grasso, M.; Soares, M.L.G. \& Tognella, M.M.P. 1994. Manguezais brasileiros, Caravelas, Estado da Bahia. Pp.324-332. In: Anais do III Simpósio de Ecossistemas da Costa Brasileira: Subsídio a um Gerenciamento Ambiental. Serra Negra, ACIESP.

Silva, C.A.R.; Lacerda, L.D. \& Rezende, C.E. 1990. Heavy metal reservoirs in a red mangrove forest. Biotropica 22(1): 339-345.

Silva, F.A.S. \& Azevedo, C.A.V. 2007. A new version of the AssistatStatistical Assistance Software. Pp. 393-396. In: Annals of the World Congress on Computers in Agriculture. Orlando, American Society of Agricultural Engineers.

Siqueira, W.G.; Braga, E.S.; Mahíques, M.M. \& Aprile, F.M. 2006. Determinação da matéria orgânica e razões $\mathrm{C} / \mathrm{N}$ e $\mathrm{C} / \mathrm{S}$ em sedimentos de fundo do estuário de Santos-SP/Brasil. Arquivos de Ciência do Mar 39: 18-27.

Souza, J.R.S.; Cohen, J.C.P.; Alvalá, R.C.S.; Costa, F.R.M.; Santos Neto, L.A. \& Abreu, S.F. 2004. Variação sazonal de temperatura e umidade em solos sob floresta, manguezal e pastagem a Amazônia. Pp. 23-26. In: Anais do XIII Congresso Brasileiro de Meteorologia, Fortaleza.

Tomlinson, P.B. 1986. The Botany of Mangroves. Cambridge, Cambridge University Press.

Twilley, R.R.; Snedaker, S.C.; Yáñez-Arancibia, A. \& Medina, E. 1995. Mangrove systems. Pp. 387-393. In: Heywood, V.H. (Org.). Global Biodiversity Assessment. Biodiversity and Ecosystem Function: Ecosystem Analyses. Cambridge, Cambridge University Press.

Wang, W.Q.; Wang, M. \& Peng, L. 2003. Seasonal changes in element contents in mangrove element retranslocation during leaf senescence. Plant Soil 252: 187-193.

Waisel, Y. 1972. Biology of halophytes. New York, Academic Press. 